\title{
Effects of Baduanjin Exercise on Cognitive Function and Cancer-related Symptoms in Women With Breast Cancer Receiving Chemotherapy: a Randomized Controlled Trial
}

\section{Xiaolin Wei}

Fudan University: Obstetrics and Gynaecology Hospital

\section{Ruzhen Yuan}

Tongji University Dongfang Hospital

\section{Juan Yang}

Shanghai University of Traditional Chinese Medicine Yueyang Hospital of Integrated Traditional Chinese Medicine and Western Medicine

\section{Wei Zheng}

Shanghai University of TCM Affiliated Longhua Hospital

\section{Yongmei Jin}

Shanghai Seventh People's Hospital

\section{Mingyue Wang}

Shanghai University of TCM: Shanghai University of Traditional Chinese Medicine

\section{Jieting Jiang}

Shanghai University of TCM: Shanghai University of Traditional Chinese Medicine

\section{Caiqin Wu ( $\nabla$ yuwuchina@aliyun.com )}

Shanghai University of TCM: Shanghai University of Traditional Chinese Medicine

\section{Kunpeng Li}

Shanghai University of TCM: Shanghai University of Traditional Chinese Medicine

\section{Research Article}

Keywords: Baduanjin exercise, Breast cancer, Cognitive function, Cancer-related symptoms, Quality of life

Posted Date: December 9th, 2021

DOI: https://doi.org/10.21203/rs.3.rs-1044265/v1

License: (9) This work is licensed under a Creative Commons Attribution 4.0 International License. Read Full License 
Version of Record: A version of this preprint was published at Supportive Care in Cancer on April 13th, 2022. See the published version at https://doi.org/10.1007/s00520-022-07015-4. 


\section{Abstract}

Purpose Cognitive decline is one of the main side effects of breast cancer patients after relevant treatment, but there is a lack of clear measures for prevention and management without definite mechanism. Moreover, postoperative patients also have a need for limb rehabilitation. Whether the cognitive benefits of Baduanjin exercise can improve the overall well-being of this breast cancer patients remains unknown. This study aims to figure out these problems in the under-researched Chinese population.

Methods This randomized controlled trial was conducted on 70 patients with breast cancer undergoing chemotherapy who were randomly assigned and allocated to (1:1) the supervised Baduanjin group (5 times/week, 30 min each time) or the control group for three months. The effects of Baduanjin exercise intervention were evaluated by outcome measures including subjective cognitive function, symptoms (fatigue, depression and anxiety) and health-related quality of life at pre-intervention (T0), 4 weeks (T1), 8 weeks (T2) and 12 weeks (T3). The collected data were analyzed by using an intention-to-treat principle and linear mixed-effects modeling.

Results Participants in the Baduanjin group had a significantly greater improvement in terms of FACT-cog $(F=14.511 ; P<0.001), \mathrm{PCl}(F=15.915 ; P<0.001), \operatorname{PCA}(F=2.767 ; P=0.047)$, and health-related quality of life $(F=8.900 ; p=0.004)$ compared with the control group over the time. The exercise-cognition relationship was significantly mediated by reduced fatigue (indirect effect: $\beta=0.132 ; 95 \% \mathrm{Cl}: 0.046$, 0.237 ) and improved anxiety (indirect effect: $\beta=-0.075 ; 95 \% \mathrm{Cl}:-0.165,-0.004$ ).

Conclusions This pilot study revealed the benefits and outlined the underlying mediating mechanism of Baduanjin exercise to the subjective cognition and health-related quality of life of Chinese breast cancer patients receiving chemotherapy. The findings provided insights into the development of public health initiatives to promote brain health and improve quality of life among breast cancer patients.

\section{Trial registration number ChiCTR 2000033152}

\section{Introduction}

Cognitive impairment in cancer patients is mostly attributed to cancer and treatment, including chemotherapeutic agents, hormone therapy, and radiation therapy, which is also known as cancer-related cognitive impairment (CRCl) [1]. $\mathrm{CRCl}$ is predominately characterized by the change of memory, processing speed, attention or concentration, and executive function [2,3], which manifests during or post-treatment with variable degrees, onset and duration. Up to $75 \%$ of cancer patients have been reported accompanied by mild to severe cognitive complaints, which can persist for months to even few years after the completion of treatment [4]. Cognitive complaints are the most frequent complication reported by breast cancer (BC) patients [5] that more than $50 \%$ of these patients following chemotherapeutic drugs exist; however, only $15 \%-25 \%$ of these cancer patients have shown poor performance in objective cognition [6]. These BC patients may experience difficulties in daily functioning, 
decision-making, multitasking and word retrieval, and treatment adherence; thereby, $\mathrm{CRCl}$ has become one of the prevalent factors that compromise the quality of life and decreased survival [7].

As the exact underlying mechanisms and etiologies of $\mathrm{CRCl}$ are still unclear, recent studies have emerged proposing several candidates. Affected cancer patients showed increased levels of C-reactive protein (CRP), tumor necrosis factor alpha, Interleukin-6 [8-11]. Meanwhile, the serum levels of Interleukin-1 receptor antagonist and Interleukin-10 were decreased [11]. At the same time, other underlying mechanisms were found, included chemotherapy-induced neurotoxicity, decreased hippocampal neurogenesis, white matter abnormalities, and increased oxidative stress [12]. In addition, pervasive fatigue and negative emotions may contribute to increased susceptibility of cognitive impairment. There was a moderate positive correlation between subjective cognitive impairment and fatigue in postmenopausal breast cancer patients [13]. The study found that BC patients undergoing systemic treatment had higher levels of fatigue and more subjective cognitive problems along with the decline of executive function [14]. The result [15] showed that the subjective cognitive function of BC patients after 6 months of chemotherapy was significantly lower than before chemotherapy, and there was a significant correlation with anxiety and depression. However, there is also a contradictory result that the association between cognitive impairment and depression didn't exist [16].

There is currently a high need for therapeutic interventions that can reduce cognitive complaints. Exercise is an established safe and effective therapy for managing adverse effects of cancer treatment, including fatigue, psychological distress, functional decline, and detrimental body composition changes [17-19]. Accumulating evidences on the exercise on positively improving cognitive function in healthy older adults and those with mild cognitive impairment or more severe neurocognitive impairment (i.e., $A D$, stroke) have sparked significant interest in the potential use of exercise as a management strategy for CRCI [20, 21]. A vast number of studies have shown that moderate-to-vigorous physical activity can certainly mitigate or ameliorate cognitive impairment in BC patients, especially in processing speed, executive function, working memory, etc. [22-24]. Two pilot studies showed that aerobic exercise improved objective cognition. One study [23] found that a 12-week exercise intervention improved the processing speed, but there was no significant improvement in self-reported cognitive function. Another study showed that a 24week supervised and home-based aerobic exercise program demonstrated similar results in postmenopausal women with early-stage breast cancer [25]. Nevertheless, one contradictory study [26] showed that aerobic exercise plus resistance training improved subjective cognitive function. Unfortunately, due to limited preclinical studies and testing, evidence-based recommendations are insufficient. Thereby, more rigorous studies need to be warranted.

Baduanjin, as a kind of exercise therapy based on mindfulness, such as Qigong and Taichi, is a combination of breathing and exercise, which is beneficial for fatigue, quality of life, and negative mood among patients $[18,19]$. Regular Baduanjin exercise can improve the cognitive function of the old people with mild cognitive impairment. Of course, there are also studies concerning medical Qigong to improve the cognitive function of BC patients. The results of study [27] showed that Qigong improved the self- 
cognition function, executive function and language fluency. In light of higher withdrawal rate and poor adherence in this study, the effect of exercise on cognitive functioning needs further explanation.

Therefore, the primary purpose of this study is to determine whether Baduanjin exercise programme is a protective measure for cognitive decline in chemotherapy-exposed patients with early-stage breast cancer. Moreover, this study aims to examine whether or not cancer-related symptoms (fatigue, depression and anxiety) and quality of life are improved and its possible association.

\section{Methods}

\section{Study design and setting}

This was a single-blinded randomized controlled trial with two study arms, including a supervised Baduanjin exercise group and a wait-list control group. Participants were recruited from the three affiliated hospitals of Shanghai University of Traditional Chinese Medicine, including Yueyang Hospital of Integrated Traditional Chinese and Western Medicine, Seventh People's Hospital, and Longhua Hospital. The outcome measures were evaluated in a quiet room in the hospital to avoid interference.

\section{Participants}

Participants were randomly assigned to an intervention group or a control group-in a 1:1 ratio using SPSS 24.0 software. Researchers screened patients who met the inclusion and exclusion criteria and provided relevant information, as well as possible benefits of the exercise and the safety. After obtaining consent, the research team examined the candidates with patients' questionnaire. The inclusion criteria were showed as follows: (1) female patients newly diagnosed with stage I to III breast cancer; (2) scheduled to receive adjuvant chemotherapy; (3) aged 40 to 75 years; (4) using WeChat; (5) sufficient fluency of the Chinese language; (6) willing to participate in the study and be randomly assigned. Exclusion criteria included participants with (1) disease recurrence or metastasis; (2) known conditions and/or diseases that impact cognition (e.g., Alzheimer's disease, dementia, or other psychological diagnoses); (3) disorders that might preclude exercise participation (e.g., fracture, arthritis); (4) current participation in mindfulness-based exercise programs (e.g., TaiChi or Qigong). Due to the nature of exercise studies, only results evaluators were blinded. More details were presented in this previous protocol of study [28].

\section{Sample size estimation}

The sample size was estimated in this study by using perceptive cognitive impairment as the main effect indicator, which refers to the results of previous study [29]. Using the $\mathrm{G}^{*}$ Power 3.1 software, 58 cases of the total sample were calculated with a test power of 0.80 . Considering the $20 \%$ dropout rate, the total sample size for this study was about 70 patients randomized to the intervention group or the control group.

\section{Study interventions}




\section{Baduanjin exercise}

Baduanjin exercise was conducted according to the standards promulgated by the General Administration of Sport in China in 2003. A professionally trained Qigong specialist uniformly trained subject researchers to learn the exercise training. Researchers then conducted on-site instruction to guide theses participants and correct their movements. Participants also were provided with video demonstration to promote daily practice. Before initiating the first cycle of chemotherapy, the patients had the first session in the hospital, then following the video workout at home. Recommended training time was 5 times a week for half an hour each time during the 12-week exercise period. The program began with stretching the joints, inhalation and exhalation for 2 min respectively, and two 12 min Baduanjin sessions, followed by 2 min of muscle relaxation exercises. Each patient's log was recorded during exercise at home, including any obstacles that affected exercise training. When the patient went to the hospital to receive the next cycle of chemotherapy, the feedback of exercise log was collected and checked.

\section{Control intervention}

Patients randomized to the control group were given a face-to-face health education. Disease-related questions raised by the patients were directly communicated or answered through WeChat online or oral communication. Patients improved the affected limb through exercises, including abduction, forward flexion, backward extension, internal rotation, and wall climbing according to the guideline and standard for the diagnosis and treatment of breast cancer in China [30]. In addition, the patients were not asked to conduct other aerobic activities. Researchers asked about their conditions twice a week during the 12 weeks.

\section{Measurement}

\section{Socio-demographic profile}

At baseline, a self-designed demographic data sheet was used to collect the social-demographic data, including age, BMI, marital status, income, education and living condition, as well as relevant disease information from electronic medical system.

\section{Outcome measurement}

The Functional Assessment of Cancer Therapy-Cognitive Function (FACT-Cog) was developed by Wagner et al [31] to assess subjective cognitive function. The Chinese versions of the FACT-Cog was used; The Multidimensional Fatigue Symptom Inventory-short form (MFSI-SF) was used to assess the fatigue of patients in multiple aspects [32]. Taiwanese scholar then revised the Chinese version of the Multidimensional Fatigue Symptom Inventory-Short Form (MFSI-SF-C) [33], which was used in this study; The Hospital Anxiety and Depression Scale (HADS) was used for the assessment of patients' anxiety and depression [34]; The Functional Assessment of Cancer Therapy-Breast (FACT-B), a 44-item self-report 
instrument was well-known and widely used to measure multidimensional quality of life (QoL) in patients with breast cancer [35].

\section{Procedure and ethical considerations}

The protection of human subject was approved by the Ethics Committee of the Seventh People's Hospital of Shanghai University of Traditional Chinese Medicine (No. 2020-7th-HIRB-011). The purpose and process were explained to potential participants. Written informed consent was obtained from each participant; each participant was assured of confidentiality and the option to decline participation or to withdraw from the trial at any time without penalty. Baseline data collection was conducted by two trained research assistants. After the pre-test data collection, the participants were randomly allocated to receive the Baduanjin exercise programme or the wait-list control group. The post-test data was collected at 4 weeks (T1), 8 weeks (T2), and 12 weeks (T3) during intervention.

\section{Data analysis}

Demographics and other characteristics were reported descriptively via the statistics software (SPSS 24.0). The means and SD were calculated for continuous normally distributed variables and medians and ranges for non-normally distributed variables. Categorical variables were presented by absolute numbers and percentages. We performed complete case analyses after describing the pattern of missing data.

According to the intention-to-treat principle, a linear mixed-effect model for repeated-measures analysis was used, in which group, time point, and the interaction between group and time point were set as fixed effects; individual patient was set as the random effects; and age was adjusted as a covariate in the model. Adherence to the Baduanjin exercise was noted. Two-sided significance level for all tests was 0.05. The PROCESS macro [36] was employed to examine the mediating effects of fatigue and anxiety on the relationship between the exercise and subjective cognition. 95\% confidence intervals for direct and indirect effects were generated via bias-corrected bootstrapping with 10,000 resamples. The nonparametric bootstrapping procedures are superior to traditional regression methods for testing the mediating effects, because the former does not make assumptions regarding the shape of the distribution of the variables or the sampling distribution [37]. Confidence intervals without zero showed significant mediation.

\section{Results}

\section{Recruitment}

As shown in the CONSORT flow diagram (Fig. 1), 107 BC patients were approached for eligibility screening. Among the 70 participants who met the selection criteria and consented to participate. During the study period, according to the results of exercise diary, 24 of the 35 patients ( $80 \%$ ) had a completion rate $>85 \%$, which could be considered as good compliance, mainly because of severe diarrhea after 
chemotherapy $(n=1)$ or voluntarily withdrew $(n=2)$. No adverse events, such as lymphedema related to exercise, occurred.

\section{Participant characteristics}

The median age of the included patients was $55(46,60)$, with the majority married and relatively loweducated (52.9\% indicating they had a junior high school education or less). $68.6 \%$ of the patients were menopausal. The majority of participants were early breast cancer patients (stage I-II) with $50 \%$ in stage II. Anthracyclines accounted for $51.4 \%$ in all chemotherapy regimens, and non-anthracyclines for $48.6 \%$; $7.1 \%$ chemotherapy regimen combined with targeted therapy; breast cancer patients with comorbidities accounted for $24.3 \%$. The demographic and clinical disease characteristics of the participants were shown in Table 1. No considerable difference was observed between groups at baseline.

\section{Effects on cognitive function}

At the 4th, 8th, and 12th weeks, FACT-Cog scores of both groups are presented in Table 2. Participants in the Baduanjin group had significantly greater improvement in their FACT-cog score compared with the wait-list control group across the baseline and post-intervention periods $(F=19.456 ; P<0.001)$. Amongst all the subscales of FACT-cog, the results were significant for $\mathrm{PCI}(F=15.915 ; P<0.001), \mathrm{PCA}(F=2.767 ; P=$ $0.047)$, the impact of cognition on the quality of life $(F=9.410 ; P=<0.001)$, and no significant difference was found in evaluation of others $(F=3.766 ; P=0.056)$.

\section{Effects on health-related quality of life}

At the 4th, 8th, and 12th weeks, FACT-B scores of both groups are presented in Table 3. Participants in the intervention group were found to have a significantly greater improvement in FACT-B score compared with the wait-list control group across the baseline and post-intervention periods ( $F=8.900 ; P=0.004)$. Amongst the FACT-B items, the mixed-effect modeling results were significant for physical well-being $(F=4.908$; $P=0.03)$, emotional well-being $(F=7.640 ; P=0.007)$ (Supplementary Table 2). No significant difference was shown in terms of social/family function, functional well-being and additional attention $(F=3.766 ; P=$ 0.056). The scores ofthe FACT-B and Constant-Murley scores in both groups of breast cancer patients were improved, but the baduanjin group was significantly better than the control group (see supplementary material).

\section{Results of the mediating analysis}

Through linear regression analysis, the FACT-cog score had a linear correlation with fatigue score $(\beta=-0.392, P<0.001)$, and it had a linear correlation with anxiety score $(\beta=0.339, P=0.001)$. There was no correlation between FACT-cog score and depression score $(\beta=-0.053, P=0.585)$. On this basis, a mediation hypothesis model of fatigue and anxiety is established. After putting fatigue and anxiety into intermediary variables through SPSS PROCESS, the result analysis of the intermediary model is shown in

Fig. 2. Exercise has a negative effect on anxiety $(\beta=-0.795, t=-2.105, P<0.05)$; exercise has a significant negative effect on fatigue $(\beta=-0.733, t=-3.319, P<0.001)$; It has a significant positive effect on cognition ( $\beta$ 
$=5.875, t=5.932, P<0.001)$; fatigue has a significant negative effect on subjective cognitive ability $(\beta=-1.618, t=-4.432, P<0.001)$. It shows that exercise can not only directly affect the improvement of subjective cognition, but also improve subjective self-cognition by reducing fatigue and anxiety. The direct effect and the mediating effect of fatigue and anxiety accounted for $50.00 \%, 18.57 \%$, and $10.59 \%$ of the total effect, respectively.

\section{Discussion}

The aim of this study is to investigate the effects of a 12-week Baduanjin exercise on cognitive function, cancer-related symptoms and quality of life in BC patients during chemotherapy, and the exercisecognition relationship. This study echoes the cognitive-enhancing benefits of other aerobic and mindbody exercise amongst breast cancer population. This study showed that exercise training can meet the treatment goal of preventing the deterioration of cognitive function among BC patients. By incorporating a mediating analysis, this study adds further evidence to indicate that cognitive benefits of physical exercise are manifested through its positive effects on two cognitive risk factors, including fatigue and anxiety. This exercise intervention may alleviate the effect of cognitive decline on the overall well-being of $B C$ patients.

\section{Cognitive benefit of exercise}

The subjective feelings of cancer patients are the most relevant measure of clinical significance, and the self-reported cognition of patients should be used as the main outcome indicator for $\mathrm{CRCl}$. The results showed that the subjective cognitive ability and $\mathrm{PCl}$ of the patients in the Baduanjin group were significantly better than those in the Baduanjin group at 8 and 12 weeks. However, no obvious significance was found between groups at the 4th week, which may be attributed to the patients in the exercise adaptation period. In addition, BC patients were in the initial postoperative recovery period, and their postures for exercise cannot be fully standardized. This was similar to the results of Myers et al [27], who divided the $\mathrm{BC}$ patients into the Qigong group, the general exercise group and the wait-list control group for 8 weeks intervention. The results showed that the PCl and PCA in the Qigong group were significantly improved compared with the wait-list control group. Another randomized controlled study [29] also conducted a 10-week medical Qigong intervention, and the results showed that the Qigong group's subjective cognitive ability and $\mathrm{PCl}$ were improved. Baduanjin exercise has a certain preventive effect on the decline of subjective cognitive ability of breast cancer patients during chemotherapy, and the continuous effect of Baduanjin intervention on cognitive ability after the end of the 12-week Baduanjin intervention has not been observed due to personnel and time constraints, which can be further observed in future large sample studies.

\section{Effects of exercise on health-related quality of life}

BC patients were attributed to cancer, cancer treatment, or combined with other diseases, developing longterm physical and psychological diseases, such as fatigue, sleep disturbance, mood, etc. Studies have shown that these patients with less education, comorbidities, and chemotherapy were more likely to 
possess poor quality of life, while lack of social support and unmet needs may lead to poor health-related life [38]. Baduanjin exercise improved quality of life among BC patients at the 8th and 12th week. In addition, this study also showed that the shoulder joint function in both groups was improved at the 8th and the 12th week, indicating that Baduanjin was beneficial to promote the recovery of the shoulder joint function, especially the active range of movement of the shoulder joint. These results were consistent with the results of Ying et al [18]. After 6 months of Baduanjin intervention, patients in the Baduanjin group had significantly improved in shoulder joint mobility, and quality of life. At the same time, another randomized controlled trial [39] also found that after 12 weeks of Baduanjin intervention, the quality of life of patients in the Baduanjin group was better than that of the control group. The anxiety and depression symptoms of the two groups were improved, but the difference between the groups was not statistically significant, and the quality of life was positively correlated with anxiety and depression. The above results indicated that Baduanjin intervention has a certain effect on improving the quality of life of breast cancer patients during chemotherapy. In addition, studies have also shown that Baduanjin had an effect on increasing BMI, relieving postoperative limb pain and lymphatic swelling [40], but significant differences in BMI and pain between the two groups was not observed in this study. The possible reason may be that most of the patients' surgical methods were lymphatic dissection, but most of them were sentinel dissection. Axillary dissection was relatively small, and some BC patients underwent breastconserving surgery, without obvious postoperative pain. Secondly, lymphedema generally appeared in 3 to 6 months after the operation, and no obvious limb swelling was found in the two groups of patients during the intervention period. Therefore, a 3-month follow-up after the end of the intervention can be done to observe the changes in future studies.

\section{Anxiety symptoms and fatigue mediated the exercise-cognition relationship}

Subjective cognitive impairment has been associated with poor physical function, anxiety, depression and fatigue, which was more likely to occur in cancer patients and even worsen [41]. Baduanjin can improve the physical function and mental health [19]. A longitudinal observational study found that fatigue played a role between physical activity and subjective cognition [42]. Another study [43] showed that the severity of cognitive impairment increased with the degree of insomnia in postmenopausal BC patients. Sleep disturbance may also be an influencing factor for $\mathrm{CRCl}$, but we did not follow up the sleep quality. In addition, Song et al [44] showed that aerobic exercise improved the quality of sleep and reduce depression in elderly people with mild cognitive impairment. Physical activity affected cognition including increasing the level of BDNF in plasma, improving metabolic function, and reducing systemic inflammation [45]. The level of BDNF was related to processing speed, increasing s significantly after aerobic exercise [46]. Future research may further explore the changes in cognitive function, such as brain tissue and its function, immunohistochemistry, etc., to contribute to the mechanism of exercise to improve cognitive function, and it needs to be further determined whether the management strategy of $\mathrm{CRCl}$ can be improved by solving fatigue, anxiety or sleep problems.

\section{Limitations}


This study has several limitations. Firstly, this study took into account the research characteristics of exercise intervention, and it was impossible to blind the subjects and Baduanjin practitioners, but only blinded the outcome assessors. Secondly, although the neuropsychological test used in the evaluation was relatively mature in clinical application, it cannot completely avoid the subjective influence of the test. Although some movements were gradually trained, the initial movements were still different from the original standardized movements, so the results could be influenced to a certain extent. In addition, the sample size is not large, and the interpretation of the results has certain limitations. A larger sample can be considered in the next study. Finally, taking into account the issue of research funding, we didn't collect the laboratory indicators related cognition, which may be included as part of mechanism research in the future, and the effects of exercise on $\mathrm{CRCl}$ can be further enriched through multidisciplinary cooperation.

\section{Conclusion}

This study presented a positive scenario of a 12-week group based on Baduanjin exercise for protection of the cognitive decline and improvement of quality of life among BC patients receiving chemotherapy. Furthermore, decreasing anxious and improving fatigue were firstly identified as part of the possible mechanisms underlying the exercise-cognition relationship. The effectiveness and feasibility of this exercise also promoted the need to increase its application against further cognitive decline in BC patients.

\section{Declarations}

\section{Acknowledgements}

We gratefully acknowledge all participants who contribute to the trial.

\section{Availability of data and material}

These data that support the findings of this study are available on request from the corresponding author. The data are not publicly available due to privacy or ethical restrictions.

\section{Code availability}

N/A.

\section{Author contribution}

Conceptualization, Methodology: [Wu Caiqin]c; Data curation, Writing-Original draft preparation: [Wei Xiaolin]; Visualization, Investigation: [Jiang Jieting], [Wang Mingyue]; Supervision: [Yang juan], [Jin Yongmei], [Zheng wei]; Software, Validation: [Yuan Ruzhen]; Writing- Reviewing and Editing: [Li Kunpeng]. All authors read and approved the final manuscript.

\section{Funding}


The trail was supported by the Nursing Subject Competence Enhancement Project of Shanghai University of Traditional Chinese Medicine (No.2020HLXK07) and Xinglin Youth Talents Training Project of Shanghai University of Traditional Chinese Medicine-Xinglin Scholar to Cai-Qin Wu.

\section{Ethics approval}

The study received ethical approval from the Ethics Committee of the Seventh People's Hospital of Shanghai University of Traditional Chinese Medicine (No. 2020-7th-HIRB-011).

\section{Consent to participate}

All participants provided informed consent prior to enrolling onto the study.

\section{Consent for publication}

All authors consent to the publication of this manuscript in Supportive Care in Cancer.

\section{Conflict of interest}

None.

\section{References}

1. Ahles TA, Saykin AJ, McDonald BC, Li Y, Furstenberg CT, Hanscom BS, Mulrooney TJ, Schwartz GN, Kaufman PA (2010) Longitudinal assessment of cognitive changes associated with adjuvant treatment for breast cancer: impact of age and cognitive reserve. J Clin Oncol 28(29): 4434-4440. https://doi: 10.1200/JC0.2009.27.0827

2. Joly F, Giffard B, Rigal O, De Ruiter MB, Small BJ, Dubois M, LeFel J, Schagen SB, Ahles TA, Wefel JS, Vardy JL, Pancre V, Lange M, Castel H (2015) Impact of Cancer and Its Treatments on Cognitive Function: Advances in Research From the Paris International Cognition and Cancer Task Force Symposium and Update Since 2012. J Pain Symptom Manage 50(6): 830-841

3. Lange M, Joly F (2017) How to Identify and Manage Cognitive Dysfunction After Breast Cancer Treatment. J Oncol Pract 13(12): 784-790

4. Falleti MG, Sanfilippo A, Maruff P, Weih L, Phillips KA (2005) The nature and severity of cognitive impairment associated with adjuvant chemotherapy in women with breast cancer: a meta-analysis of the current literature. Brain Cogn 59(1): 60-70

5. Schmidt JE, Beckjord E, Bovbjerg DH, Low CA, Posluszny DM, Lowery AE, Dew MA, Nutt S, Arvey SR, Rechis $R$ (2016) Prevalence of perceived cognitive dysfunction in survivors of a wide range of cancers: results from the 2010 LIVESTRONG survey. J Cancer Surviv 10(2): 302-311

6. Lange M, Licaj I, Clarisse B, Humbert X, Grellard JM, Tron L, Joly F (2019) Cognitive complaints in cancer survivors and expectations for support: Results from a web-based survey. Cancer Med 8(5): 2654-2663. https://doi: 10.1002/cam4.2069 
7. Robb C, Boulware D, Overcash J, Extermann M (2010) Patterns of care and survival in cancer patients with cognitive impairment. Crit Rev Oncol Hematol 74(3): 218-224

8. Cheung YT, Ng T, Shwe M, Ho HK, Foo KM, Cham MT, Lee JA, Fan G, Tan YP, Yong WS, Madhukumar P, Loo SK, Ang SF, Wong M, Chay WY, Ooi WS, Dent RA, Yap YS, Ng R, Chan A (2015) Association of proinflammatory cytokines and chemotherapy-associated cognitive impairment in breast cancer patients: a multi-centered, prospective, cohort study. Ann Oncol 26(7): 1446-1451

9. Kesler S, Janelsins M, Koovakkattu D, Palesh O, Mustian K, Morrow G, Dhabhar FS (2013) Reduced hippocampal volume and verbal memory performance associated with interleukin- 6 and tumor necrosis factor-alpha levels in chemotherapy-treated breast cancer survivors. Brain Behav Immun 30 Suppl: S109-116

10. Pierce BL, Ballard-Barbash R, Bernstein L, Baumgartner RN, Neuhouser ML, Wener MH, Baumgartner KB, Gilliland FD, Sorensen BE, McTiernan A, Ulrich CM (2009) Elevated biomarkers of inflammation are associated with reduced survival among breast cancer patients. J Clin Oncol 27(21): 3437-3444. https://doi: 10.1200/JC0.2008.18.9068

11. van Vulpen JK, Schmidt ME, Velthuis MJ, Wiskemann J, Schneeweiss A, Vermeulen RCH, Habermann N, Ulrich CM, Peeters PHM, van der Wall E, May AM, Steindorf K (2018) Effects of physical exercise on markers of inflammation in breast cancer patients during adjuvant chemotherapy. Breast Cancer Res Treat 168(2): 421-431

12. Mounier N, Abdel-Maged A, Wahdan S, Gad A, Azab S (2020) Chemotherapy-induced cognitive impairment (CICI): An overview of etiology and pathogenesis. Life Sci 258: 118071. https://doi: 10.1016/j.Ifs.2020.118071

13. Schilder CM, Seynaeve C, Linn SC, Boogerd W, Beex LV, Gundy CM, Nortier JW, van de Velde CJ, van Dam FS, Schagen SB (2012) Self-reported cognitive functioning in postmenopausal breast cancer patients before and during endocrine treatment: findings from the neuropsychological TEAM sidestudy. Psycho-oncology 21(5): 479-487. https://doi: 10.1002/pon.1928

14. Menning S, de Ruiter MB, Veltman DJ, Boogerd W, Oldenburg HS, Reneman L, Schagen SB (2017) Changes in brain activation in breast cancer patients depend on cognitive domain and treatment type. PLoS One 12(3): e0171724. https://doi: 10.1371/journal.pone.0171724

15. Biglia N, Bounous VE, Malabaila A, Palmisano D, Torta DM, D'Alonzo M, Sismondi P, Torta R (2012) Objective and self-reported cognitive dysfunction in breast cancer women treated with chemotherapy: a prospective study. Eur J Cancer Care (Engl) 21(4): 485-492. https://doi: 10.1111/j.1365-2354.2011.01320.x

16. De Rosa N, Della Corte L, Giannattasio A, Giampaolino P, Di Carlo C, Bifulco G (2021) Cancer-related cognitive impairment (CRCI), depression and quality of life in gynecological cancer patients: a prospective study. Arch Gynecol Obstet. https://doi: 10.1007/s00404-020-05896-6

17. Lu Y, Qu HQ, Chen FY, Li XT, Cai L, Chen S, Sun YY (2019) Effect of Baduanjin Qigong Exercise on Cancer-Related Fatigue in Patients with Colorectal Cancer Undergoing Chemotherapy: A Randomized Controlled Trial. Oncol Res Treat 42(9): 431-439. https://doi: 10.1159/000501127 
18. Ying W, Min Q, Lei T, Na Z, Li L, Jing L (2019) The health effects of Baduanjin exercise (a type of Qigong exercise) in breast cancer survivors: A randomized, controlled, single-blinded trial. Eur J Oncol Nurs 39: 90-97. https://doi: 10.1016/j.ejon.2019.01.007

19. Zou L, Pan Z, Yeung A, Talwar S, Wang C, Liu Y, Shu Y, Chen X, Thomas GA (2018) A Review Study on the Beneficial Effects of Baduanjin. J Altern Complement Med 24(4): 324-335. https://doi: $10.1089 / \mathrm{acm} .2017 .0241$

20. Tao J, Liu J, Chen X, Xia R, Li M, Huang M, Li S, Park J, Wilson G, Lang C, Xie G, Zhang B, Zheng G, Chen L, Kong J (2019) Mind-body exercise improves cognitive function and modulates the function and structure of the hippocampus and anterior cingulate cortex in patients with mild cognitive impairment. Neurolmage Clin 23: 101834. https://doi: 10.1016/j.nicl.2019.101834

21. Xia R, Qiu P, Lin H, Ye B, Wan M, Li M, Tao J, Chen L, Zheng G (2019) The Effect of Traditional Chinese Mind-Body Exercise (Baduanjin) and Brisk Walking on the Dorsal Attention Network in Older Adults With Mild Cognitive Impairment. Front Psychol 10: 2075. https:// doi: 10.3389/fpsyg.2019.02075

22. Ehlers DK, Aguinaga S, Cosman J, Severson J, Kramer AF, McAuley E (2017) The effects of physical activity and fatigue on cognitive performance in breast cancer survivors. Breast Cancer Res Treat 165(3): 699-707. https://doi: 10.1007/s10549-017-4363-9

23. Hartman SJ, Nelson SH, Myers E, Natarajan L, Sears DD, Palmer BW, Weiner LS, Parker BA, Patterson RE (2018) Randomized controlled trial of increasing physical activity on objectively measured and self-reported cognitive functioning among breast cancer survivors: The memory \& motion study. Cancer 124(1): 192-202. https://doi: 10.1002/cncr.30987

24. Marinac CR, Godbole S, Kerr J, Natarajan L, Patterson RE, Hartman SJ (2015) Objectively measured physical activity and cognitive functioning in breast cancer survivors. J Cancer Surviv 9(2): 230-238. https://doi: 10.1007/s11764-014-0404-0

25. Campbell K, Kam J, Neil-Sztramko S, Liu Ambrose T, Handy T, Lim H, Hayden S, Hsu L, Kirkham A, Gotay C, McKenzie D, Boyd L (2018) Effect of aerobic exercise on cancer-associated cognitive impairment: A proof-of-concept RCT. Psycho-oncology 27(1): 53-60. https://doi: 10.1002/pon.4370

26. Galvao DA, Taaffe DR, Spry N, Joseph D, Newton RU (2010) Combined resistance and aerobic exercise program reverses muscle loss in men undergoing androgen suppression therapy for prostate cancer without bone metastases: a randomized controlled trial. J Clin Oncol 28(2): 340-347. https://doi: 10.1200/jco.2009.23.2488

27. Myers JS, Mitchell M, Krigel S, Steinhoff A, Boyce-White A, Van Goethem K, Valla M, Dai J, He J, Liu W, Sereika SM, Bender CM (2019) Qigong intervention for breast cancer survivors with complaints of decreased cognitive function. Support Care Cancer 27(4): 1395-1403. https://doi: 10.1007/s00520018-4430-8

28. Wei XL, Yuan RZ, Jin YM, Li S, Wang MY, Jiang JT, Wu CQ, Li KP (2021) Effect of Baduanjin exercise intervention on cognitive function and quality of life in women with breast cancer receiving 
chemotherapy: study protocol of a randomized controlled trial. Trials 22(1): 405. https://doi: 10.1186/s13063-021-05355-w

29. Oh B, Butow PN, Mullan BA, Clarke SJ, Beale PJ, Pavlakis N, Lee MS, Rosenthal DS, Larkey L, Vardy J (2012) Effect of medical Qigong on cognitive function, quality of life, and a biomarker of inflammation in cancer patients: a randomized controlled trial. Support Care Cancer 20(6): 12351242. https://doi: 10.1007/s00520-011-1209-6

30. Jiang ZF, Li JB (2020) [Development of guidelines and clinical practice for breast cancer]. Zhonghua Wai Ke Za Zhi 58(2): 85-90. https:// doi: 10.3760/cma.j.issn.0529-5815.2020.02.002

31. Wagner LI, Sweet J, Butt Z, Lai JS, Cella D (2009) Measuring patient self-reported cognitive function: Development of the Functional Assessment of Cancer Therapy-Cognitive Function Instrument. J Support Oncol 7: W32-W39

32. Stein KD, Martin SC, Hann DM, Jacobsen PB (1998) A multidimensional measure of fatigue for use with cancer patients. Cancer Pract 6(3): 143-152. https:// doi: 10.1046/j.15235394.1998.006003143.x

33. Pien LC, Chu H, Chen WC, Chang YS, Liao YM, Chen CH, Chou KR (2011) Reliability and validity of a Chinese version of the Multidimensional Fatigue Symptom Inventory-Short Form (MFSI-SF-C). J Clin Nurs 20(15-16): 2224-2232. https://doi: 10.1111/j.1365-2702.2010.03691.x

34. Zigmond A, Snaith R (1983) The hospital anxiety and depression scale. Acta psychiatrica Scandinavica 67(6): 361-370. https://doi: 10.1111/j.1600-0447.1983.tb09716.x

35. Brady MJ, Cella DF, Mo F, Bonomi AE, Tulsky DS, Lloyd SR, Deasy S, Cobleigh M, Shiomoto G (1997) Reliability and validity of the Functional Assessment of Cancer Therapy-Breast quality-of-life instrument. J Clin Oncol 15(3): 974-986. https://doi: 10.1200/jco.1997.15.3.974

36. Hayes AF, Rockwood NJ (2017) Regression-based statistical mediation and moderation analysis in clinical research: Observations, recommendations, and implementation. Behav Res Ther 98: 39-57. https://doi: 10.1016/j.brat.2016.11.001

37. Preacher KJ, Hayes AF (2004) SPSS and SAS procedures for estimating indirect effects in simple mediation models. Behav Res Methods Instrum Comput 36(4): 717-731. https://doi: 10.3758/bf03206553

38. Ho P, Gernaat S, Hartman M, Verkooijen H (2018) Health-related quality of life in Asian patients with breast cancer: a systematic review. BMJ open 8(4): e020512. https://doi: 10.1136/bmjopen-2017020512

39. Liu P, You J, Loo W, Sun Y, He Y, Sit H, Jia L, Wong M, Xia Z, Zheng X, Wang Z, Wang N, Lao L, Chen J (2017) The efficacy of Guolin-Qigong on the body-mind health of Chinese women with breast cancer: a randomized controlled trial. Qual Life Res 26(9): 2321-2331. https://doi: 10.1007/s11136-0171576-7

40. Panchik D, Masco S, Zinnikas P, Hillriegel B, Lauder T, Suttmann E, Chinchilli V, McBeth M, Hermann W (2019) Effect of Exercise on Breast Cancer-Related Lymphedema: What the Lymphatic Surgeon Needs to Know. J Reconstr Microsurg 35(1): 37-45. https://doi: 10.1055/s-0038-1660832 
41. Lange M, Joly F, Vardy J, Ahles T, Dubois M, Tron L, Winocur G, De Ruiter MB, Castel H (2019) Cancerrelated cognitive impairment: an update on state of the art, detection, and management strategies in cancer survivors. Ann Oncol 30(12): 1925-1940. https://doi: 10.1093/annonc/mdz410

42. Phillips S, Lloyd G, Awick E, McAuley E (2017) Relationship between self-reported and objectively measured physical activity and subjective memory impairment in breast cancer survivors: role of self-efficacy, fatigue and distress. Psycho-oncology 26(9): 1390-1399. https://doi: 10.1002/pon.4156

43. Liou KT, Ahles TA, Garland SN, Li QS, Bao T, Li Y, Root JC, Mao JJ (2019) The Relationship Between Insomnia and Cognitive Impairment in Breast Cancer Survivors. JNCI Cancer Spectr 3(3): pkz041. https://doi: 10.1093/jncics/pkz041

44. Song D, Yu D (2019) Effects of a moderate-intensity aerobic exercise programme on the cognitive function and quality of life of community-dwelling elderly people with mild cognitive impairment: A randomised controlled trial.Int J Nurs Stud 93: 97-105. https://doi: 10.1016/j.jnurstu.2019.02.019

45. Shahid M, Kim J (2020) Exercise May Affect Metabolism in Cancer-Related Cognitive Impairment. Metabolites 10(9). https://doi: 10.3390/metabo10090377

46. Szuhany K, Bugatti M, Otto M (2015) A meta-analytic review of the effects of exercise on brainderived neurotrophic factor. J Psychiatr Res 60: 56-64. https://doi: 10.1016/j.jpsychires.2014.10.003

\section{Tables}

Table 1 Comparison of demographic and baseline characteristics. 


\begin{tabular}{|c|c|c|c|c|c|}
\hline Characteristic & $\begin{array}{l}\text { Overall } \\
(n=70)\end{array}$ & $\begin{array}{l}\text { Intervention } \\
\text { group }(n=35)\end{array}$ & $\begin{array}{l}\text { Control group } \\
(n=35)\end{array}$ & $t / z / \chi^{2}$ & $P$ \\
\hline Age, y, median (range) \# & $55 \rrbracket 46 \rrbracket 60 \rrbracket$ & $52 \rrbracket 43 \rrbracket 60 \rrbracket$ & $55 \rrbracket 50 \rrbracket 62 \rrbracket$ & -0.912 & 0.362 \\
\hline $\mathrm{BMI}$, mean $\pm S D$ & $23.06 \pm 2.54$ & $22.86 \pm 2.55$ & $23.26 \pm 2.56$ & -0.645 & 0.521 \\
\hline Educational level, n (\%)\# & & & & -0.704 & 0.481 \\
\hline Middle school or less & $37 \rrbracket 52.9 \% \rrbracket$ & $17 \rrbracket 24.3 \% \rrbracket$ & $20 \rrbracket 28.6 \% \rrbracket$ & & \\
\hline High school & $19 \llbracket 27.1 \% \rrbracket$ & $10 \bigotimes 14.3 \% \rrbracket$ & $9 \otimes 12.9 \% \rrbracket$ & & \\
\hline College & $13 \rrbracket 18.6 \% \bigotimes$ & $8 \otimes 11.4 \% \bigotimes$ & $5 \bigotimes 7.1 \% \bigotimes$ & & \\
\hline Postgraduate & $1 \otimes 1.4 \% \bigotimes$ & $0 \otimes 0 \% \bigotimes$ & $1 \otimes 1.4 \% \bigotimes$ & & \\
\hline Marriage, n (\%) & & & & 0.355 & 0.837 \\
\hline Single & $3 \rrbracket 4.3 \% \rrbracket$ & $1 \rrbracket 1.4 \% \% \rrbracket$ & $2 \rrbracket 2.9 \% \rrbracket$ & & \\
\hline Married & $65 \rrbracket 92.9 \% \rrbracket$ & $33 \rrbracket 47.1 \% \rrbracket$ & $32 \rrbracket 45.7 \% \rrbracket$ & & \\
\hline Widowed & $2 \rrbracket 2.9 \% \rrbracket$ & $1 \rrbracket 1.4 \% \bigotimes$ & $1 \rrbracket 1.4 \% \rrbracket$ & & \\
\hline Employment status, n (\%) & & & & 1.228 & 0.541 \\
\hline Retired & $27 \rrbracket 38.6 \% \rrbracket$ & $13 \rrbracket 18.6 \% \rrbracket$ & $14 \rrbracket 20.0 \% \rrbracket$ & & \\
\hline Employed & $20 \rrbracket 28.6 \% \rrbracket$ & $12 \bigotimes 17.1 \% \bigotimes$ & $8 \otimes 11.4 \% \bigotimes$ & & \\
\hline Unemployed & $23 \rrbracket 32.9 \% \rrbracket$ & $10 \rrbracket 14.3 \% \rrbracket$ & $13 \rrbracket 18.6 \% \rrbracket$ & & \\
\hline $\begin{array}{l}\text { Monthly household Income } \\
\text { बRMB囚, n (\%)\# }\end{array}$ & & & & -0.977 & 0.328 \\
\hline$\bigotimes 1000$ & $2 \rrbracket 2.9 \% \rrbracket$ & $1 \rrbracket 1.4 \% \bigotimes$ & $1 \rrbracket 1.4 \% \rrbracket$ & & \\
\hline $1000 \sim 2000$ & $4 \rrbracket 5.7 \% \rrbracket$ & $3 \rrbracket 4.3 \% \rrbracket$ & $1 \rrbracket 1.4 \% \rrbracket$ & & \\
\hline $2000 \sim 3000$ & $17 \rrbracket 24.3 \% \rrbracket$ & $10 \rrbracket 14.3 \% \rrbracket$ & $7 \rrbracket 10.0 \% \rrbracket$ & & \\
\hline $3000 \sim 5000$ & $18 \rrbracket 27.1 \% \rrbracket$ & $8 \rrbracket 11.4 \% \rrbracket$ & $10 \otimes 15.7 \% \rrbracket$ & & \\
\hline$\nabla 5000$ & $29 \llbracket 40 \% \rrbracket$ & $13 \otimes 18.6 \% \bigotimes$ & $16 \rrbracket 21.4 \% \rrbracket$ & & \\
\hline Menopausal status, n (\%) & & & & 1.061 & 0.303 \\
\hline Pre-menopausal & $22 \llbracket 31.4 \% \rrbracket$ & $13 \otimes 18.6 \% \bigotimes$ & $9 \rrbracket 12.9 \% \rrbracket$ & & \\
\hline Post-menopausal & $48 \rrbracket 68.6 \% \rrbracket$ & $22 \rrbracket 31.4 \% \bigotimes$ & $26 \llbracket 37.1 \% \rrbracket$ & & \\
\hline BC stage, $\mathrm{n}(\%)^{\#}$ & & & & -0.202 & 0.840 \\
\hline ( & $27 \rrbracket 38.6 \% \rrbracket$ & $13 \rrbracket 18.6 \% \rrbracket$ & $14 \llbracket 20.0 \% \rrbracket$ & & \\
\hline प & $35 \rrbracket 50 \% \rrbracket$ & $18 \rrbracket 25.7 \% \rrbracket$ & $17 \rrbracket 23.4 \% \rrbracket$ & & \\
\hline
\end{tabular}




\begin{tabular}{|c|c|c|c|c|c|}
\hline प & $8 \unrhd 11.4 \% \rrbracket$ & $4 \llbracket 5.7 \% \rrbracket$ & $4 \llbracket 5.7 \% \rrbracket$ & & \\
\hline Type of surgery, $n(\%)$ & & & & 3.530 & 0.171 \\
\hline Lumpectomy & $12 \bigotimes 17.1 \% \rrbracket$ & $6 \rrbracket 8.6 \% \rrbracket$ & $6 \rrbracket 8.6 \% \rrbracket$ & & \\
\hline Quadrantectomy & $23 \rrbracket 32.9 \% \rrbracket$ & $8 \otimes 11.4 \% \rrbracket$ & $15 \rrbracket 21.4 \% \rrbracket$ & & \\
\hline Mastectomy & $35 \rrbracket 50 \% \rrbracket$ & $21 \varangle 30.0 \% \rrbracket$ & $14 \rrbracket 20.0 \% \rrbracket$ & & \\
\hline $\begin{array}{l}\text { Chemotherapy regimens, } \mathrm{n} \\
(\%)\end{array}$ & & & & 3.660 & 0.056 \\
\hline Anthracycline-based & $36 \rrbracket 51.4 \% \rrbracket$ & $22 \varangle 31.4 \% \rrbracket$ & $14 \rrbracket 20.0 \% \rrbracket$ & & \\
\hline Taxane-based & $34 \rrbracket 48.6 \% \rrbracket$ & $13 \rrbracket 18.6 \% \bigotimes$ & $21 \otimes 30.0 \% \rrbracket$ & & \\
\hline $\begin{array}{l}\text { Combined targeted therapy, } \\
\text { n (\%) }\end{array}$ & & & & $<0.001$ & 1.000 \\
\hline No & $65 \rrbracket 92.9 \% \rrbracket$ & $33 \llbracket 47.1 \% \% \rrbracket$ & $32 \rrbracket 45.7 \% \rrbracket$ & & \\
\hline Yes & $5 \bigotimes 7.1 \% \rrbracket$ & $2 \rrbracket 2.9 \% \rrbracket$ & $3 \llbracket 4.3 \% \rrbracket$ & & \\
\hline Comorbidity, n (\%) & & & & 0.324 & 0.569 \\
\hline Yes & $17 \rrbracket 24.3 \% \rrbracket$ & $9 \otimes 12.9 \% \rrbracket$ & $8 \otimes 11.4 \% \bigotimes$ & & \\
\hline No & $53 \rrbracket 75.7 \% \rrbracket$ & $26 \rrbracket 37.1 \% \rrbracket$ & $27 \rrbracket 38.6 \% \% \rrbracket$ & & \\
\hline \multicolumn{6}{|l|}{ Patient-reported outcomes } \\
\hline FACT-Cog, mean (SD) & & $124.34 \pm 8.77$ & $123.31 \pm 8.37$ & 0.502 & 0.617 \\
\hline $\mathrm{PCl}$ & & $70.11 \pm 5.05$ & $69.23 \pm 5.08$ & 0.731 & 0.467 \\
\hline PCA & & $25.60 \pm 1.88$ & $25.94 \pm 1.75$ & -0.790 & 0.432 \\
\hline FACT impact on QOL & & $13.86 \pm 1.54$ & $13.37 \pm 1.57$ & -1.562 & 0.118 \\
\hline $\begin{array}{l}\text { FACT comments from } \\
\text { others }\end{array}$ & & $14.77 \pm 1.42$ & $14.77 \pm 1.37$ & -0.155 & 0.877 \\
\hline $\begin{array}{l}\text { Fatigue MFSI-SF-C, mean } \\
\text { (SD) }\end{array}$ & & $5.01 \pm 1.92$ & $4.77 \pm 1.98$ & 0.513 & 0.610 \\
\hline Anxiety HASD-A, mean (SD) & & $5.63 \pm 2.24$ & $5.97 \pm 2.78$ & -0.231 & 0.817 \\
\hline $\begin{array}{l}\text { Depression HASD-D, mean } \\
\text { (SD) }\end{array}$ & & $6.14 \pm 2.56$ & $6.11 \pm 2.61$ & 0.046 & 0.963 \\
\hline QOL FACT-B, mean (SD) & & & & & \\
\hline
\end{tabular}

Abbreviations: BC, breast cancer; SD, standard deviation; FACT-Cog, Functional Assessment of Cancer Therapy-Cognitive Function; PCA, perceived cognitive abilities; $\mathrm{PCl}$, perceived cognitive impairments; 
MFSI-SF-C, Chinese version of the Multidimensional Fatigue Symptom Inventory-Short Form; HASD, Hospital Anxiety and Depression Scale; QOL, quality of life.

${ }^{\#} \mathrm{P}$ values for between-group differences were calculated using the Mann - Whitney $\mathrm{U}$ test.

Table 2 The comparison of subjective cognition between the intervention and control groups. 


\begin{tabular}{|c|c|c|c|c|c|c|c|c|}
\hline & \multirow[t]{2}{*}{$\begin{array}{l}\text { Intervention } \\
\text { group }\end{array}$} & \multirow[t]{2}{*}{$\begin{array}{l}\text { Control } \\
\text { group }\end{array}$} & \multicolumn{2}{|c|}{ Time effect } & \multicolumn{2}{|c|}{ Group effect } & \multicolumn{2}{|c|}{$\begin{array}{l}\text { Group*time } \\
\text { effect }\end{array}$} \\
\hline & & & $\mathrm{F}$ & $P$ & $\mathrm{~F}$ & $P$ & $F$ & $P$ \\
\hline \multicolumn{9}{|c|}{$\begin{array}{l}\text { FACT- } \\
\text { cog } \\
\text { scores }\end{array}$} \\
\hline TO & $124.34 \pm 8.77$ & $123.31 \pm 8.37$ & 19.456 & $<0.001$ & 14.511 & $<0.001$ & 23.589 & $<0.001$ \\
\hline T1 & $121.29 \pm 8.57$ & $117.51 \pm 8.15$ & & & & & & \\
\hline T2 & $122.56 \pm 7.65$ & $114.31 \pm 7.05$ & & & & & & \\
\hline T3 & $125.50 \pm 7.62$ & $113.00 \pm 6.94$ & & & & & & \\
\hline \multicolumn{9}{|c|}{$\mathrm{PCl}$} \\
\hline TO & $70.11 \pm 5.05$ & $69.23 \pm 5.08$ & 15.915 & $<0.001$ & 15.789 & $<0.001$ & 19.842 & $<0.001$ \\
\hline T1 & $68.11 \pm 4.96$ & $65.94 \pm 5.12$ & & & & & & \\
\hline T2 & $68.97 \pm 4.54$ & $64.11 \pm 4.01$ & & & & & & \\
\hline T3 & $70.69 \pm 4.28$ & $63.46 \pm 4.16$ & & & & & & \\
\hline \multicolumn{9}{|c|}{ PCA } \\
\hline TO & $25.60 \pm 1.88$ & $25.94 \pm 1.75$ & 2.767 & 0.047 & 6.261 & 0.015 & 5.631 & 0.002 \\
\hline T1 & $24.80 \pm 2.00$ & $24.71 \pm 1.58$ & & & & & & \\
\hline T2 & $25.21 \pm 1.72$ & $24.20 \pm 1.76$ & & & & & & \\
\hline T3 & $25.82 \pm 1.68$ & $23.91 \pm 1.76$ & & & & & & \\
\hline \multicolumn{9}{|c|}{ evaluation of others } \\
\hline TO & $14.77 \pm 1.42$ & $14.77 \pm 1.37$ & 3.766 & 0.056 & 9.359 & $<0.001$ & 11.229 & $<0.001$ \\
\hline T1 & $14.83 \pm 1.18$ & $14.29 \pm 1.43$ & & & & & & \\
\hline $\mathrm{T} 2$ & $14.75 \pm 1.06$ & $14.06 \pm 1.33$ & & & & & & \\
\hline T3 & $15.22 \pm 0.73$ & $14.03 \pm 1.18$ & & & & & & \\
\hline \multicolumn{9}{|c|}{ Impact on QoL } \\
\hline TO & $13.86 \pm 1.54$ & $13.37 \pm 1.57$ & 9.410 & $<0.001$ & 17.428 & $<0.001$ & 7.548 & $<0.001$ \\
\hline T1 & $13.54 \pm 1.70$ & $12.57 \pm 1.48$ & & & & & & \\
\hline T2 & $13.62 \pm 1.60$ & $11.94 \pm 1.57$ & & & & & & \\
\hline T3 & $13.77 \pm 1.86$ & $11.49 \pm 1.90$ & & & & & & \\
\hline
\end{tabular}


Table 3 The comparison of quality of life between the intervention and control groups. 


\begin{tabular}{|c|c|c|c|c|c|c|c|c|}
\hline & \multirow[t]{2}{*}{$\begin{array}{l}\text { Intervention } \\
\text { group }\end{array}$} & \multirow[t]{2}{*}{$\begin{array}{l}\text { Control } \\
\text { group }\end{array}$} & \multicolumn{2}{|c|}{ Time effect } & \multicolumn{2}{|c|}{ Group effect } & \multicolumn{2}{|c|}{$\begin{array}{l}\text { Group*time } \\
\text { effect }\end{array}$} \\
\hline & & & $\mathrm{F}$ & $P$ & $\mathrm{~F}$ & $P$ & $\mathrm{~F}$ & $P$ \\
\hline \multicolumn{9}{|c|}{ Fatigue } \\
\hline TO & $5.01 \pm 1.92$ & $4.77 \pm 1.98$ & 45.453 & $<0.001$ & 7.723 & 0.007 & 4.627 & 0.004 \\
\hline $\mathrm{T} 1$ & $2.87 \pm 1.30$ & $3.64 \pm 1.83$ & & & & & & \\
\hline T2 & $2.06 \pm 1.21$ & $3.42 \pm 1.68$ & & & & & & \\
\hline T3 & $1.95 \pm 0.85$ & $2.99 \pm 1.51$ & & & & & & \\
\hline \multicolumn{9}{|c|}{ Anxiety } \\
\hline T0 & $5.63 \pm 2.24$ & $5.97 \pm 2.78$ & 78.453 & $<0.001$ & 3.714 & 0.058 & 1.255 & 0.291 \\
\hline $\mathrm{T} 1$ & $2.37 \pm 2.38$ & $2.94 \pm 2.84$ & & & & & & \\
\hline $\mathrm{T} 2$ & $0.83 \pm 0.98$ & $2.34 \pm 2.56$ & & & & & & \\
\hline T3 & $0.76 \pm 1.23$ & $1.60 \pm 2.19$ & & & & & & \\
\hline \multicolumn{9}{|c|}{ Depression } \\
\hline T0 & $6.14 \pm 2.56$ & $6.11 \pm 2.61$ & 58.321 & $<0.001$ & 4.581 & 0.036 & 3.590 & 0.016 \\
\hline $\mathrm{T} 1$ & $3.80 \pm 2.35$ & $3.83 \pm 2.18$ & & & & & & \\
\hline T2 & $1.57 \pm 1.08$ & $3.26 \pm 2.55$ & & & & & & \\
\hline T3 & $1.25 \pm 1.27$ & $2.66 \pm 2.21$ & & & & & & \\
\hline \multicolumn{9}{|c|}{ Quality of life } \\
\hline \multicolumn{9}{|c|}{ FACT-B scores } \\
\hline TO & $93.55 \pm 8.49$ & $93.89 \pm 10.70$ & 74.219 & $<0.001$ & 8.900 & 0.004 & 8.248 & $<0.001$ \\
\hline $\mathrm{T} 1$ & $100.57 \pm 7.30$ & $99.89 \pm 10.20$ & & & & & & \\
\hline $\mathrm{T} 2$ & $110.87 \pm 8.53$ & $101.66 \pm 8.23$ & & & & & & \\
\hline T3 & $113.26 \pm 6.25$ & $106.17 \pm 6.72$ & & & & & & \\
\hline \multicolumn{9}{|c|}{ physiological status } \\
\hline TO & $19.89 \pm 2.45$ & $20.37 \pm 3.02$ & 12.023 & $<0.001$ & 4.908 & 0.030 & 2.757 & 0.045 \\
\hline $\mathrm{T} 1$ & $22.00 \pm 2.59$ & $21.26 \pm 3.41$ & & & & & & \\
\hline T2 & $23.69 \pm 5.08$ & $21.63 \pm 2.33$ & & & & & & \\
\hline T3 & $23.33 \pm 1.75$ & $21.74 \pm 2.43$ & & & & & & \\
\hline
\end{tabular}




\begin{tabular}{|lllllllll|}
\hline T0 & $22.12 \pm 1.27$ & $21.46 \pm 1.82$ & 38.915 & $<0.001$ & 1.798 & 0.184 & 0.769 & 0.513 \\
\hline T1 & $19.26 \pm 3.01$ & $19.23 \pm 2.70$ & & & & & & \\
\hline T2 & $21.22 \pm 2.38$ & $20.43 \pm 2.42$ & & & & & & \\
\hline T3 & $22.12 \pm 1.27$ & $21.46 \pm 1.82$ & & & & & & \\
\hline T0 & $16.71 \pm 2.86$ & $17.20 \pm 2.80$ & 46.561 & $<0.001$ & 7.640 & 0.007 & 5.633 & 0.001 \\
\hline T1 & $19.77 \pm 1.65$ & $19.29 \pm 2.79$ & & & & & & \\
\hline T2 & $21.37 \pm 1.73$ & $19.51 \pm 2.59$ & & & & & & \\
\hline T3 & $21.84 \pm 1.68$ & $19.71 \pm 2.05$ & & & & & & \\
\hline functional & & & & & & & & \\
\hline status & & & & & & & \\
\hline T0 & $10.23 \pm 3.34$ & $10.91 \pm 4.49$ & 82.301 & $<0.001$ & 3.811 & 0.055 & 5.020 & 0.002 \\
\hline T1 & $14.86 \pm 2.86$ & $15.11 \pm 3.51$ & & & & & & \\
\hline T2 & $18.70 \pm 2.48$ & $15.86 \pm 2.96$ & & & & & & \\
\hline T3 & $19.26 \pm 3.06$ & $17.49 \pm 2.56$ & & & & & & \\
\hline additional attention & & & & & & & & \\
\hline T0 & $24.60 \pm 3.01$ & $23.94 \pm 3.59$ & 6.180 & $<0.001$ & 2.354 & 0.130 & 1.659 & 0.179 \\
\hline T1 & $24.69 \pm 3.46$ & $25.00 \pm 3.30$ & & & & & & \\
\hline T2 & $26.71 \pm 2.92$ & $25.77 \pm 2.56$ & & & & & & \\
\hline T3 & $26.71 \pm 2.92$ & $25.77 \pm 2.56$ & & & & & & \\
\hline
\end{tabular}

\section{Supplementary Tables}

Supplementary Tables are not available with this version.

\section{Figures}




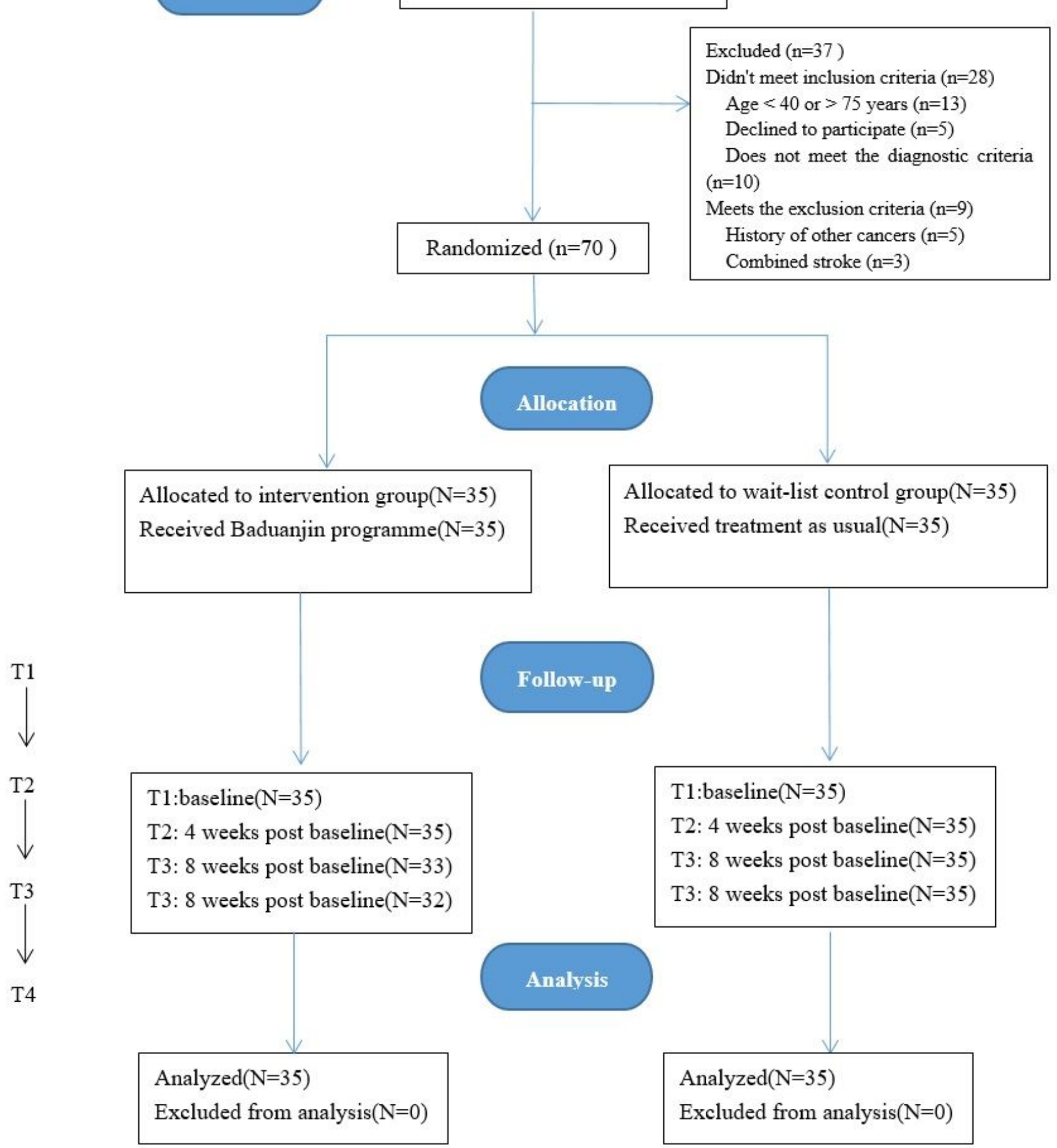

\section{Figure 1}

The flow diagram of the recruitment and randomization processes for the current study. 


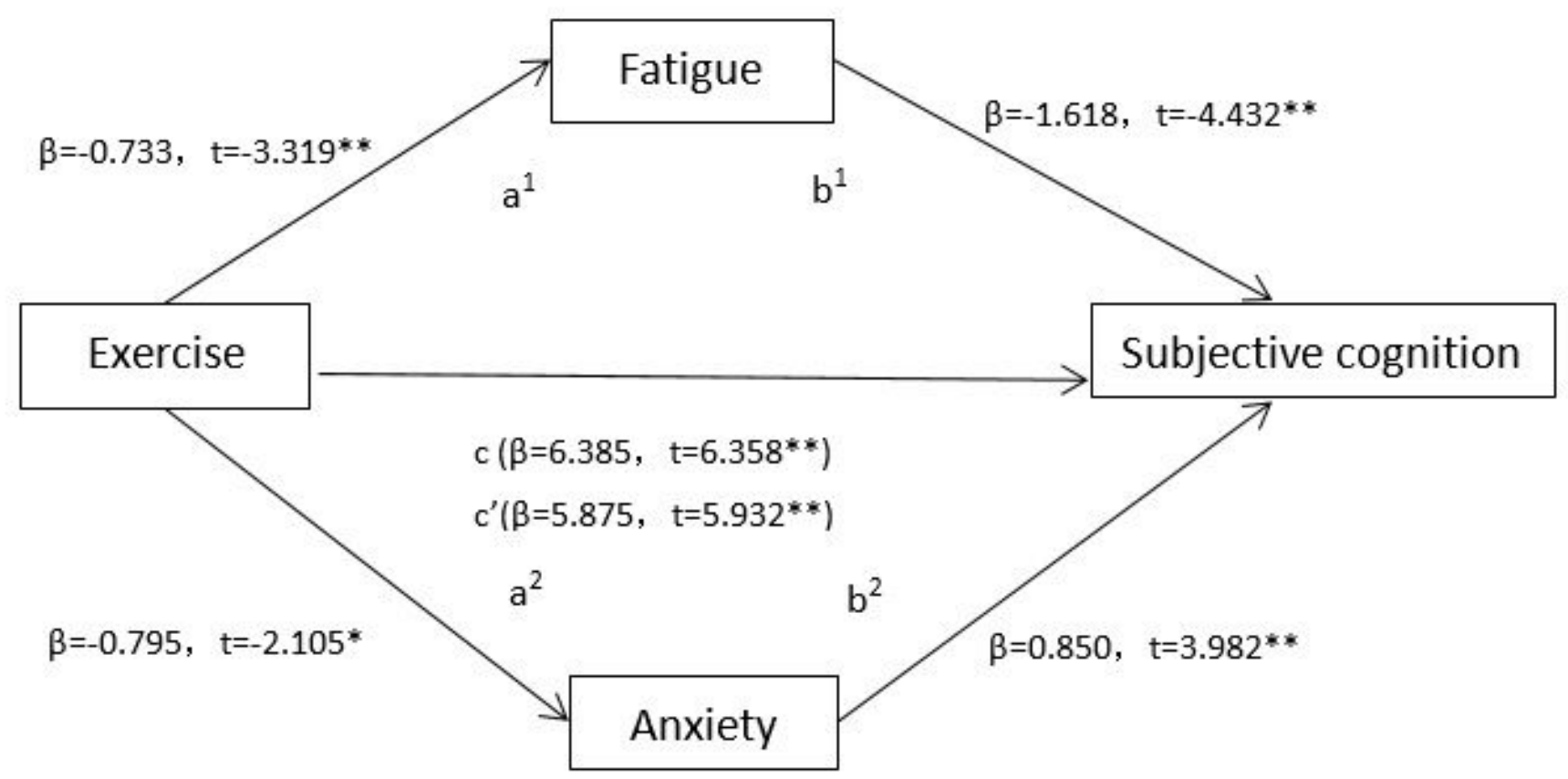

Figure 2

Mediating roles of fatigue and anxiety in the relationship between the Baduanjin exercise and the cognitive changes.

\section{Supplementary Files}

This is a list of supplementary files associated with this preprint. Click to download.

- Supplementarymaterial.docx 